\title{
FISIONOMIA DAS SAVANAS DE RORAIMA, BRASIL ${ }^{1}$
}

\author{
Izildinha Souza Miranda ${ }^{2}$, Maria Lúcia $\mathrm{Absy}^{3}$
}

\begin{abstract}
RESUMO - Quarenta e cinco parcelas de 0,15 ha $(10 \times 150 \mathrm{~m})$ distribuidas nas savanas de Roraima foram analisadas quanto a fisionomia e sua relação com as características edáficas do solo superficial. Foram utilizadas medidas quantitativas de altura, freqüência, área basal e densidade dos elementos lenhosos e medidas qualitativas de distribuição espacial e grau de cobertura arbustivo/arbóreo. As parcelas foram classificadas em quatro tipos fisionômicos (campo limpo, campo sujo, campos cerrados e savana parque) com caracteristicas estruturais bem definidas. Foram encontradas 55 familias, 137 gêneros e 267 espécies, sendo, 195 espécies herbáceas e 71 espécies lenhosas. Entre as lenhosas, $51 \%$ são arbóreas, $32 \%$ arbustivas e $17 \%$ subarbustivas. O estrato herbáceo é o mais rico em sua flora, com 41 familias, 98 gêneros e 207 espécies. Porém, apenas 18 espécies foram encontradas em mais de $20 \%$ das parcelas. Oitenta por cento das parcelas classificadas como campos limpos foram encontradas sobre solos arenosos, $81 \%$ dos campos sujos sobre solos argilosos, $64 \%$ dos campos cerrados sobre solos arenosos e todas as savanas parques foram encontradas sobre solos de areia barrenta. Entretanto, os tipos fisionômicos não possuem relações significativas com a maioria dos nutrientes analisados; apenas os teores de $\mathrm{Al}^{+++}$, na camada superficial $(0-10 \mathrm{~cm}$ de profundidade) foram significativamente diferentes entre os tipos fisionômicos. Essa diferença significativa deve-se principalmente às diferenças encontradas entre os teores de $\mathrm{Al}^{+++}$dos campos limpos e campos sujos e entre os campos limpos e campos cerrados.
\end{abstract}

Palavras-chaves: Fisionomias, Savanas, Roraima.

\section{Physiognomy of the Savannas of Roraima, Bazil}

ABSTRACT - Forty five plots of 0,15ha (10 X 150m) distributed in the savannas of Roraima were analysed for physiognomy and their relationship to the edaphic characteristics of the surface soil. Quantitative measures of height, frequency, basal area and density of woody elements and qualitative measures of spatial distribution and \% cover of tree/shrubs were used. The plots were classified in to four physiognomic types (grassland, tree and shrub savanna, woodland savanna and park savanna) each with very defined structural characteristics. The survey found 267 species, belonging to 55 families, 137 genera, 195 herbaceous species and 71 woody species. Among the woody, $51 \%$ were tree , $32 \%$ shrub and $17 \%$ dwarf shrubs. The herbaceous stratum is the richest, with 41 families, 98 genera and 207 species. However, only 18 species were found in more than $20 \%$ of the plots. Eighty percent of the plots classified as grassland were found on sandy soils, $81 \%$ of the tree and shrub savanna on clay soils, $64 \%$ of the woodland savanna on sandy soils and all the parks savannas were found on soils of loamy sand. However, the physiognomic types did not possess significant relationships with most of the analyzed nutrients; only the levels of $\mathrm{Al+++}$, in the surface layer $(0-10 \mathrm{~cm}$ depth), were significantly different among the physiognomic types. That significant difference was due mainly to the differences found between the levels of $\mathrm{Al+++}$ of the grassland and tree and shrub savanna and between the grassland and woodland savannas.

Key-words: Physiognomy, Savannas, Roraima.

\footnotetext{
'Parte da Tese de Doutorado de Izildinha S. Miranda.

${ }^{2}$ Bolsista da CAPES, INPA/CPBO. Endereço atual: Faculdade de Ciências Agrárias do Pará DCF, Av. Tancredo Neves s/n, CP 917, 66077-530, Belém, PA, Brasil.

${ }^{3}$ Instituto Nacional de Pesquisas da Amazônia, INPA/CPBO, CP 478, 69011-970 Manaus, AM, Brasil.
} 


\section{INTRODUÇÃO}

As savanas de Roraima, maior área de savana da Amazônia Brasileira, são classificadas nas categorias de "non-flooded grassland savannas" de Prance (1996), "seasonal savannas" de Sarmiento \& Monasterio (1975) e "llanos-type" de Huber (1982).

Nas classificações brasileiras, Camargo et al. (1976) e Goodland \& Ferri (1979) consideram as savanas de Roraima como cerrado, vegetação encontrada no planalto central brasileiro. Eiten (1977; 1983) distingue todas as savanas da Amazônia como um tipo de vegetação diferente do cerrado, devido a pobreza de espécies e indivíduos arbóreos encontrados nas savanas amazônicas, embora o autor destaca a alta similaridade fisionômica encontrada entre as savanas amazônicas e os cerrados do planalto central brasileiro.

Veloso et al., (1975) consideram dois tipos de savanas em Roraima, com sete categorias diferentes: savanas (graminosa, arbórea aberta e parque) e savanas-estépicas (graminosa, arbórea aberta, arbórea densa e parque), mas os autores conjectura sobre a existência das savana-estépicas. A separação entre savana e savana-estépica foi basicamente geográfica, as savanas limitam-se ao norte com a sub-região da superfície dissecada (vulcânica) do alto Surumu, ao sul e a oeste com o contato Floresta/Floresta estacional e a leste ultrapassa os limites da fronteira Brasil-Guiana. As savanas- estépicas limitam-se ao sul com a região das savanas e ao norte com a sub-região montanhosa do Parima (floresta tropical de altitude) e a oeste também ultrapassa os limites da fronteira Brasil-Guiana. Veloso et al., (1991) classificam as savanas de Roraima em savana arborizada (campo cerrado) e savana-estépica, mas nada falam sobre as savanasestépicas.

Vanzolini \& Carvalho (1991) propõem a denominação de lavrado (termo popular para as savanas de Roraima) para diferenciá-las dos demais cerrados brasileiros, em função da grande abundância de Cyperaceae nessas savanas, além da baixa diversidade floristica.

Os fatores edáficos são considerados os mais importantes na determinação da heterogeneidade encontradas nas savanas da América do Sul, principalmente a fertilidade (Eiten, 1972; Goodland \& Pollard, 1973; Queiroz Neto, 1982; Montgomery \& Askew, 1983; Ratter \& Dargie, 1992) e as variações da saturação d'água, causadas pela flutuação do nivel do lençol freático (Goldsmith, 1974; Huber, 1982; Oliveira \& Martins, 1986; Furley \& Ratter, 1988; Oliveira et al., 1989). Entretanto, fogo, herbivoria e distúrbios antrópicos possuem papéis diferentes em cada região, sendo considerados como determinantes secundários desses ecossistemas (Gibbs et al., 1983; Medina, 1987; Borhidi, 1988; Pagano et al., 1989; Durigan et al., 1994; Hoffmann, 1996). 
Nas savanas de Roraima, nenhum trabalho quantitativo foi feito a fim de caracterizar as fisionomias e correlacioná-las com fatores ambientais. Deste modo, este trabalho tem por objetivo estudar 45 comunidades vegetais das savanas de Roraima, visando, principalmente (1) identificar os tipos fisionômicos das savanas de Roraima e (2) relacionar as fisionomias encontradas com fatores edáficos.

\section{ÁREA DE ESTUDO}

As savanas de Roraima localizam-se no Estado de Roraima, na parte mais setentrional do Brasil, com uma área de $230.104 \mathrm{~km}^{2}$. Essas savanas estendem-se até as Guianas, na região do Rio Rupununi, onde elas tem a mesma fisionomia, por isso elas são denominadas "savanas RoraimaRupununi” (Pires \& Prance, 1985).

Localizam-se sobre o Planalto do Amazonas-Orenoco e sobre a Depressão da Amazônia Setentrional. O planalto trata-se do grande divisor de águas das bacias hidrográficas dos Rios Orinoco e Amazonas (Gatto, 1991). As savanas encontram-se nos níveis inferiores desse planalto, a altitudes que variam entre 400 e $800 \mathrm{~m}$, numa extensa área montanhosa, de origem pré-cambriana pertencentes ao Grupo Roraima, Formação Surumu e Granodiorito Serra do Mel (Franco et al., 1975).

Na Depressão da Amazônia Setentrional, as savanas encontram-se sobre o Pediplano Rio Branco-Rio Negro pertencentes à Formação Boa Vista. O relevo é predominantemente aplainado, altitude entre 80 e $160 \mathrm{~m}$, onde pode-se encontrar diversos afloramentos de rochas, constituindo pequenos "inselbergs", que podem ter altitudes de 400 a $500 \mathrm{~m}$. Apresenta uma topografia de ondulações pouco acentuadas, regionalmente conhecida por "tesos", onde ocorrem geralmente blocos ferruginosos (Franco et al., 1975). Nessa região a drenagem é constituída por igarapés na sua maioria intermitentes, marcados por Mauritia flexuosa (que forma os buritizais), como nas veredas do Brasil Central (Pires \& Prance, 1985). Também ocorrem pequenas depressões formando lagos de forma geralmente circular, isolados ou parcialmente drenados para igarapés (Veloso et al., 1975).

O estudo climático da região das savanas é baseado numa única estação meteorológica localizada em Boa Vista, havendo uma certa dificuldade em caracterizar toda a área das savanas (Ambtec, 1994). O clima típico dessas savanas é Tropical Monçônico (Awi de Köppen) com altas temperaturas médias durante o ano e estação seca bem acentuada. (Veloso et al., 1975). A temperatura média anual é de $24^{\circ} \mathrm{C}$ com variações anuais entre 26 e $29^{\circ} \mathrm{C}$. A umidade relativa anual média é de $85 \%$. A precipitação média anual é $1500 \mathrm{~mm}$, podendo variar em até $1000 \mathrm{~mm}$ se considerar os anos mais secos e anos mais chuvosos (Ambtec, 1994).

O regime sazonal de precipitação define duas estações, seca e chuvosa, que revela um padrão inverso do que se verifica nas áreas meridionais da 
Amazônia (Nimer, 1991). As estações são bem definida: estação chuvosa, entre abril e agosto/setembro, quando os totais mensais de chuva variam em média entre 150 e $350 \mathrm{~mm}$; e, estação seca, entre outubro e março, quando os totais mensais são geralmente inferiores a $50 \mathrm{~mm}$, resultando em deficiência hidríca (Nimer, 1991; Ambtec, 1994).

As savanas de Roraima situamse sobre vários tipos de solos, segundo Corrêa et al. (1975). A região bem ao norte situam-se principalmente sobre Solos Litólicos Distróficos e afloramentos rochosos, com ou sem areias quartzosas distróficas e lateritas hidromórficas. Na região da Formação Boa Vista encontram-se quatro principais tipos de solos: Entre o médio Rio Surumu e médio Rio Parimé encontram-se solos de (1) Laterita Hidromórfica Distrófica associados a planassolo eutrófico e areias quartzosas hidromóficas distróficas e (2) Planassolo Eutrófico. $\mathrm{Na}$ área central encontram-se (3) Latossolos Amarelo distrófico associados a areias quartzosas distróficas e laterita hidromórfica distrófica. Ao leste e sul das savanas, encontram-se (4) Latossolos Vermelho Amarelo associados a solos concrecionados lateríticos, latossolo vermelho escuro distrófico e laterita hidromórfica distrófica (Corrêa et al., 1975).

\section{MÉTODOS}

A físionomia foi analisada através da composição floristica lenhosa e herbácea de 45 parcelas de 0,15 ha distribuidas ao longo das rodovias existentes na região de savanas de Roraima. Foram utilizadas as medidas quantitativas de altura, freqüência relativa $(\mathrm{Fr}=100 \mathrm{X}$ número de parcelas presentes/ número total de parcelas), área basal e densidade dos elementos lenhosos. A distribuição espacial dos elementos lenhosos foi determinada em categorias qualitativas: agrupados em moitas ou dispersos. O grau de cobertura arbustivo/arbóreo foi determinado, segundo Sarmiento (1984), em três categorias: (1) cobertura menor que $2 \%$, (2) cobertura entre 2 e $15 \%$ e (3) cobertura maior que $15 \%$. A classificação fisionômica foi baseada nos critérios estabelecidos por Sarmiento (1984) para as savanas Neotropicais.

Os solos da camada superficial $(0-10 \mathrm{~cm}$ de profundidade) foram analisados quanto aos teores de Alumínio $\left(\mathrm{Al}^{+++}\right)$, Cálcio $\left(\mathrm{Ca}^{++}\right)$, Magnésio $\left(\mathrm{Mg}^{++}\right)$, Potássio $\left(\mathrm{K}^{+}\right)$, Sódio $\left(\mathrm{Na}^{+}\right)$, Fósforo disponível (P), Matéria Orgânica (MO), argila e areia. Os métodos e análises sobre os solos das parcelas estudadas encontram-se em Miranda (1998).

Regressão e teste de Tukey foram usados para relacionar os tipos fisionômicos com as características edáficas e verificar diferenças entre os tipos de solos (Zar, 1984).

\section{RESULTADOS}

Nas 45 parcelas amostradas foram encontrados 55 familias, 137 gêneros e 267 espécies (Tab. 1), sendo, 
Tabela 1. Espécies coletadas nas savanas de Roraima. Típos Biológicos: $\mathrm{E}=$ ervas, $\mathrm{SB}=$ subarbustos, $\mathrm{AB}=$ arbusto, $\mathrm{A}=$ árvore, $\mathrm{T}=$ trepadeira, $\mathrm{P}=$ parasita, $\mathrm{HP}=$ hemiparasita.

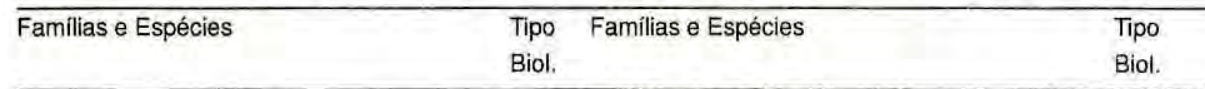

\section{MONOCOTILEDONEAS}

AGAVACEAE

Fourcroya sp.

\section{ALISMATACEAE}

Echinodorus cf. tenella (Mart.) Buch.

\section{AMARYLLIDACEAE \\ Curculigo scorzonerifolia Baker \\ Amaryllis cf. belladona Linn.}

\section{ARACEAE}

Caladium bicolor Engler

\section{CYPERACEAE}

Abildgaardia ovata

Bulbostylis conifera Kunth.

Bulbostylis consaguinea

Bulbostylis junciformis C.B.Clarke

Bulbostylis juncoides Kunth. ex Osten.

Bulbostylis lanata DC.

Bulbostylis paradoxa (Spreng.)

Bulbostylis sphaerocephala C.B.Clarke

Bulbostylis tenuifolia Macbr,

Bulbostylis truncata

Cyperus aggregatus

Cyperus aristatus Rottb.

Cyperus haspan Boeckl.

Cyperus laetus Kunth.

Cyperus laxus

Cyperus sphacelatus

Cyperus surinamensis Boeckl.

Eleocharis filiculmis

Eleocharis interstincta (Vahl) Roem. Schult. Kyllinga odorata Boeckl.

Lagenocarpus guianensis Pfeiff.

Lagenocarpus rigidus Pfeiff.

Rhynchelytrum repens (Willd.) C.E. Hubb.

Rhynchospora albo-marginata Kunth.

Rhynchospora armerioides

Rhynchospora barbata Boeckl.

Rhynchospora cephalotes Vitt.

Rhynchospora pr. emaciata (Nees) Boack.

Rhynchospora globosa Kunth.

Rhynchospora hirsuta

Rhynchospora holoschoenoides (L.C. Rick.)

Rhynchospora nervosa Kunth.

Rhynchospora sect. Pauciflorae

Rhynchospora subplumosa C.B.Clarke
ERIOCAULACEAE

Indeterminada 1

Indeterminada 2

E

E

E

E

\section{HAEMODORACEAE}

Schiekia orinocensis (Kunth) Meisner subsp. orinocensis Maas \& Stoel.

\section{IRIDACEAE}

E Cipura paludosa Aubl.

E Elentherine sp.

Sisyrinchium minutiflorum Klatt.

E

MAYACACEAE

Mayaca Kunthii Seub.

ORCHIDACEAE

Habenaria cf. staminodiata Schltt.

POACEAE

Andropogon angustatus (J.S. Presl) Steud E

Andropogon bicornis $\mathrm{L}$.

Andropogon fastigiatus Sw.

Andropogon leucostachyus HBK.

Andropogon selloanus Hack.

Andropogon virgatus Desv.

Aristida gibbosa (Nees) Kunth

Aristida recurvata Kunth

Aristida sp.

Axonopus aureus Beauv.

Axonopus conduplicatus G.A. Black

Axonopus sp.

Dactyloctenium aegyptium (L.) Beauv.

Echinolaena inflexa (Poir) Chase

Eleusine indica (L.) Gaert.

Elyonorus sp.

Elyonorus muticus (Spreng.) OKtze. E

Eragrostis sp.

Leptocoryphum lanatum (Kunth) Nees

Mesosetum chasae Luces

Mesoetum loliforme (Hochst) Chase

Otachyrium succisum (Swallen) Sendulshy

\& Soderstom

Panicum lanciflorum

Panicum laxum Haek.

Panicum ligulare

Panicum parvifolium

Panicum tricoides

Panicum sp.

Paspalum carinatum
E

E

E

E

E

E

E

E

E

E

E

E

E

E

E

E

E 
Tab, 1 - Continuação

Rhynchospora velutina Pilger

Scleria bracteata Pfeiff.

Scleria hirtella Boeckl.

Scleria pteroba Kunth.

Scleria scabra

Scleria setaceae Kunth.

Scleria tenella Kunth.

Sacciolepis angustissima (Steud.) Kunth

Sacciolepis myurus Chase

Schizachyrium hertiflorum Nees

Schizachyrium microstachyum (Desv. ex Hamil) Ros. Arr. \& Izag

Schizachyrium sanguineum (Retz) Alst

Schizachyrium tenerum Nees

Schizachyrium sp.

Setaria parviflora (Poir) Kerguelen

Setaria pauciflora

Setaria sp.

Sporobolus cubensis Hitchc.

Sporobolus indicus (L.) R. Brown

Sporobolus sp.

Thrasya petrosa (Trin.) Chase

\section{DICOTILEDONEAS}

\section{ACANTHACEAE}

Dipteracanthus angustifolia (Nees) Brem.

\section{ANACARDIACEAE}

Tapirira guianensis Aubl.

Indeterminada

\section{ANNONACEAE}

Annona jahnii Saff.

Xylopia aromatica

\section{APOCYNACEAE}

Himatanthus articulatus

Mandevila cf. pavoii (DC.) Woods

Odontadenia geminata (R. \& S.) Muell Arg.

\section{ASCLEPIADACEAE}

Blepharodon nitidum (Vall.) Macbr.

\section{ASTERACEAE}

Acanthospermum australe Kuntze Centratherum cf. punctatum Cass. Rolandra fruticosa (L.) Kuntze Indeterminada
Paspalum ceresia (Kuntze) Chase

Paspalum gardnerianum Nees

Paspalum lancifiorum Trin.

Paspalum plicatum Michx

Paspalum pulchellum

Pennisetum polystachyum (L.) Schult

Plagiantha tenella Renv.

Plagiantha sp.

Thrasya thrasyoides (Trin.) Chase

Trachypogon spicatus (L.f.) Kuntze

Trachypogon plumosus $\mathrm{L}$.

Trachypogon canescens

Trachypogon sp.

Urochloa brizantha (Hochst ex A. Rich) R. Webster.

\section{PONTEDERIACEAE}

Eichornia azurea (Sw.) Kunth

E

SMILACACEAE

Smilax sp.

\section{XYRIDACEAE}

Xyris laxifolia Mart.

\section{E}

Cassia cultrifolia H.B.K.

Cassia curvifolia Vog.

Cassia desvauxii Calladon

Cassia diphylla L.

E Cassia flexuosa L.

Cassia hispidula Vahl.

Cassia nictitans L.

A Cassia obtusifolia L.

Cassia sp.

Copaifera cf. coriacea Mart.

A Peltogyne campestris Cucke

A Peltogyne paniculata Benth.

$S+D 117$ wartzia cf. laurifolia Benth.

E

E

E

E

E

\section{A CARYOPHYLLACEAE}

L Polycarpea corymbosa Lam.

\section{CHRYSOBALANACEAE}

Hirtella ciliata Mart. \& Zucc.

L Hirtella paniculata Sw.

\section{CLUSIACEAE}

E Clusia cf. renggerioides PI. et Tr.

E Vismia cayannensis (Jacq.) Pers.

$A B \quad V i s m i a$ guianensis (Aubl.) Choisy

$\mathrm{AB}$ Vismia japurensis Reichardt.

Vismia macrophylla H.B.K.

E
E
E
E
A
A
A
E
E

$A$
$A$
A
A
A
A
A
A
E
E
E


Tab. 1 - Continuação

\section{BIGNONIACEAE}

Jacaranda obtusifolia var. obtusifolia

$A B$

CONNARACEAE

Xylopheagma sumannianum (Oktze) Sand. A

Indeterminada

\section{BORAGINACEAE}

Cordia corymbosa (L.) Don.

Cordia multispicata Cham.

Heliotropium helophyllum Mart.

\section{CACTACEAE}

\section{Cactus sp1}

Cactus sp2

Cereus paraensis Hub.

Cereus sp.

Melocactus neryi K. Sch.

\section{CAESALPINACEAE}

Cassia calyciodes DC. ex Callad

Jacquemontia cf. sphaerostigma (Cav.)

Rusby

Merremia cissioides (Lam.) Hallier

Merremia aturenesis (H.B.K.) Hallier

Merremia sp.

Quamoclit cf. rodriguesii Falcao

\section{CYRILLIACEAE}

Cyrilla racemifiora $\mathrm{L}$.

\section{DILLENIACEAE}

Curatella americana L.

Davilla aspera (Aubl.) Benoist

\section{ERYTHROXYLACEAE \\ Erythroxylum cf. faetidum Plowman \\ Erythroxylum suberosum St. Hil. \\ Erythroxylum subracemosum Turcz.}

\section{EUPHORBIACEAE}

Euphorbia cf. cotinifolia L.

Euphorbia sp.

Mabea cf. pulcherrima Mull. Arg.

Phyllanthus caroliniensis Walt.

Phyllanthus stipulatus (Raf.) Webster.

Sebastiana bidentada (Mart.) Pax

Chamaesyce cf. hirta (L.) Millsp.

Cnidoscolus urens (L.) Arthur

Croton sp.

Euphorbia brasiliensis Lam.

Euphorbia cf. caecorum Mart.

Schultesia benthamiana Klotsch ex Griseb.

Schultesia aff. brachyptera Cham.

\section{CONVOLVULACEAE}

SB Anisea sp.

SB Bonamia sp

E Evolvulus filipes Mart.

Evolvulus cf. glomeratus Nees \& Mart.

Evolvulus cf. saxifragus

E Evolvulus sericeus Sw.

E Evolvulus tenesis Mart.

E Evolvulussp.

E Ipomoea cf. assarifolia (Desr.) Roem. \&

E Schl.

Jacquemontia agrestes (Mart. ex Meish) E Meicn.

E Jacquemontia tamnifolia Griseb.

L

E Stylosanthes guianensis (Aubl.) Sw. var. E gracilis (H.B.K.) Vog.

E Tephrosia cinerea Pers.

E Tephrosia cf. leptostachya DC.

E Tephrosia nitens Benth

E Sommeringia semperflarens Mart.

Zornia diphylla (L.) Pers.

A FLACOURTIACEAE

Casearia grandiflora Camb.

A

Casearia sylvestris Swartz var. lingua

A (Camb.) Eichler

AB Casearia uimifolia Vahl ex Ventenat

A

\section{GENTIANACEAE}

A

Coutoubea ramosa Aubl. var. ramosa

E Benth.

A Curtia tenuiflora (Aubl.) Knobl. E

Irlbachia alata (Aubl.) Maas. E

Irlbachia cf. caerulescens (Aubl.) Griseb. E

A

E HUMIRIACEAE

A Humiria balsamifera (Aubl.) St. Hil.

A

E

E HYDROPHYLLACEAE

E Hydrolea spinosa L.

$A B$

$E$

\section{AB LAMIACEAE}

AB Hyptis atrorubus Poit.

E Hyptis brevipes Poit. E

E Hyptis cf. hirsuta Kunth E

E Hyptis cf. obtusifolia Presl. E

E Hyptis recurvata Poit. AB 
Tab. 1 - Continuação

\section{FABACEAE}

Acosmius nitens (Vog.) Yokevtev.

Aeschynomene brasiliana DC.

Aeschynomene histrix Poir

Aeschynomene paniculata Willd. ex Vog

Aeschynomene cf. sensitiva Sw.

Andira riveriana Arroyo

Bowdichia virgilioides H.B.K.

Centrosema angustifolium (H.B.K.) Benth.

Crotalaria maypurensis H.B.K.

Desmodium adscendens (Sw.) DC.

Desmodium asperum (Poir) Desv.

Desmodium intortum (Mill.) Urban.

Desmodium sclerophyllum Benth.

Desmodium sp.

Derris floribunda Benth

Dioclea guianensis Benth

Eriosema crinitum (H.B.K.) E. Mey.

Eriosema heterophyllum Benth.

Eriosema sp.

Galactia jussiaeana Kunth

Indigofera hirsuta L.

Indigofera lespedesioides H.B.K.

Indigofera cf. suffroticosa Miller

Ormosia smithii Rudd.

Pterocarpus cf. rohrii Vahl

Rhynchosia macrocarpa Benth.

Rhynchosia sp.

Stylosanthes angustifolia Vog.

MALVACEAE

Sida cf. acuta Burm.

Sida cf. cordifolia L.

Sida cf. glaziovii K. Sch.

Sida linearifolia St. Hil.

Sida cf. mantiana St. Hil.

Sida rhombifolia L.

\section{MELASTOMATACEAE}

Acisanthera limnobios (DC.) Trina.

Acisanthera uniflora (Vahl.) Gleason

Clidemia cf. rubra (Aubl.) Mart.

Comolia purpurea miq. Sens. Cogn.

Comolia veronicaefolia Benth

Desmoncelis villosa (Aubl.) Naud.

Macairea pachyphylla Benth.

Miconia ciliata

Miconia rofescens (Aubl.) DC.

Miconia rubiginosa (Bonpl.) DC.

Miconia argyrophylla DC.

Pterolepis glomerata (Rottb.) Miq.

Rhynchanthera hispida Naud.

Rhynchanthera grandiflora (Aubl.) DC.
Hyptis suaveolens Poit.

E

A LAURACEAE

E Cassytha filifornis $\mathrm{L}$.

E

Endlicheria arunciflora Mes.

$P$

A

\section{LENTIBULARIACEAE}

A Genlisea repens Benj.

A

Utricularia erectiflora St. Hil.

Utricularia fimbriata H.B.K.

Utricularia flaccida DC.

LORANTHACEAE

Phthirusa adunca (G.F.W. Mey) Maguire

LOGANIACEAE

Antonia ovata

$A B$ Spigelia anthelmia $\mathrm{L}$.

LYTHRACEAE

E Cuphea antisyphilitica H.B.K.

Cuphea ramulosa Mart.

MALPIGHIACEAE

Banisteriopsis muricata (Cavanicata)

Cuatrecasas

Byrsonima coccolobifolia

A Byrsonima crassifolia (L.) H.B.K.

$A B$ Byrsonima schomburgkiana A

$L \quad$ Byrsonima verbascifolia $A B$

E Byrsonimasp. $\quad A B$

OCHNACEAE

$E$

Sauvagesia sprengelli St. Hil.

E

E

OPILIACEAE

E Indeterminada

A

AB PASSIFLORACEAE

E Passifiora cf. candellei Tr.

Passiflora cf, faetida L.

$E$

A POLYGALACEAE

$\mathrm{AB} \quad$ Polygala sp1

$A B \quad P o l y g a l a s p 2$

AB Polygalasp3 E

A Polygalasp4

$\mathrm{E}$

AB PORTULACACEAE

$A B$ Portulaca oleracea $L$.
HP

E

A

E

E

A

$\mathrm{E}$

$E / A B$

$A B$

L

E

E

E

E

P

A

E

A

A

A

$A B$

$A B$

E

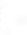


Tab. 1 - Continuação

Rhynchanthera cf. sirrulata (Rich.) DC.

Tibouchina aspera Aubl.

\section{MENISPERMACEAE}

Cissampelos ovalifolia DC.

\section{MENYANTHACEAE}

Nymphoides humboldtiana (H.B.K.) OKZE

\section{MIMOSACEAE}

Acacia cf. polyphylla DC.

Anadenanthera peregrina

Mimosa camporum Benth.

Mimosa debiles H.B.K.

Mimosa microcephala Humb.

Mimosa ct. niederleinii Burk.

Mimosa pudica L.

\section{MORACEAE}

Dorstenia brasiliensis Lam.

MYRSINACEAE

Myrsine guianensis (Aubl.) O. Ktze

\section{MYRTACEAE}

Calycolpus goetheanus (DC.) Berg.

Eugenia cf. coarensis DC.

Eugenia punicifolia (H.B.K.) DC.

Eugenia sp.

Psidium guianense $\mathrm{Sw}$.

Psidium sp.

\section{NYMPHEACEAE}

Nymphaea rudgeana G.F.W. Meyer

\section{OCHNACEAE}

Ouratea cf. schomburgkii

Ouratea rigida Engl.

SCROPHULARIACEAE

Bacopa cirphyllifolia (Benth.) Pennell.

Bacopa salzmanii (Benth.) Wettst.

Buchnera palustris (Aubl.) Spreng.

Buchnera rosea H.B.K.

Conobea aquatica Aubl.

Lindernia crustaces (L.) F. Von Muell.

Lindernia cf. dubia Pennell.

Lindernia sp.

\section{SOLANACEAE}

Cistrum sp.

Solanum monachophyilum Dunal.

Solanum subinerme Jacq.

\section{STERCULIACEAE}

Melochia cf. anomala Griseb.

Melochia arenosa Benth.

Melochia hirsuta Cav.

Melochia melissaefolia Benth.

Melochia villosa (Mill.) Fawc. et Rend.
E

$A B$

\section{PROTEACEAE}

Roupala montana Aubl.

E

\section{RUBIACEAE}

Borreria cf. alata

E

E

Borreriacapitata

Borreria ocimoides (Burm. f.) DC.

Borreria suaveolens G.F.W. Meyer

A Borreria verticilata (L.) G.F.W. Meyer

Chomelia cf. tenuiffora Benth

Chomelia sp.

AB Diodia hyssopifolia Cham. et Sclecht.

$A B$ Farameasp.

AB Genipa americana $L$.

E Pagamea guianensis Aubl.

Pagamea coriacea Spruce ex Benth.

Palicourea rigida H.B.K.

E

Pallasia standleyana Klotzch.

Perama hirsuta Aubl.

Psychotria sp.

A Psyllocarpus psyllocarpoides (Sucre) Kirke

Randia armata Sw.

Randia formosa (Jack.) Schum. $\quad A B$

A Randia hebecarpa AB

AB Sipanea pratensis Aubl. E

$A B$ Tocoyena formosa AB

$A B$ Tocoyena neglecta N.E. Brown. AB

$A B$

AB SAPINDACEAE

Cupania polydonta Radlk.

Paulinia leiocarpa Griseb.

E Mataybasp.

A

A

A

SAPOTACEAE

A Manilkarasp.

A

TILIACEAE

E Apeiba albiflora Duck.

E Carchorus hirtus L.

E

A

TRIGONIACEAE

Trigonia vilosa Aubl.

A

TURNERACEAE

Piriqueta cf. cistoides G.F.W. meyer

Piriqueta duartiana (Comb.) Urban.

Piriqueta sp.

A Turnera cf. scabra Millspaugh

A Turnera ulmifolia L.

$\mathrm{AB}$ Turnera $\mathrm{sp}$.

\section{VERBENACEAE}

E Aegiphylla integrifolia (Jacq.) Jacks. A

E Lippia microphylla Cham. AB

AB Stachytarpheta cf. elatiar Schult. E

E Vitex polyana Cham. A

E Vitex schomburgkiana Schum. A 
195 espécies herbáceas e 71 espécies lenhosas. Entre as lenhosas, $51 \%$ são arbóreas, $32 \%$ arbustivas e $17 \%$ subarbustivas (Tab. 2). O estrato herbáceo é o mais rico em sua flora, com 41 famílias, 98 gêneros e 207 espécies. Porém, apenas 18 espécies foram encontradas em mais de $20 \%$

Tabela 2. Número de Familias, gêneros e espécies coletados nas savanas de Roraima.

\begin{tabular}{lcccc}
\hline & Ervas & Subarbustos & Arbustos & Árvores \\
\hline Familias & 31 & 08 & 12 & 22 \\
Gêneros & 96 & 11 & 18 & 30 \\
Espécies & 195 & 12 & 23 & 36 \\
N.. de parcelas & 45 & 45 & 35 & 34 \\
\hline
\end{tabular}

das parcelas.

Quatro tipos fisionômicos, com caracteristicas estruturais bem definidas, foram identificados nas savanas de Roraima: savana graminosa (campo limpo), savana aberta (campo sujo), savana arborizada (campos cerrados) e savana parque (Tab. 3).

As savanas graminosas com Byrsonima verbascifolia, além de dominar a paisagem entre Boa Vista e Alto Alegre, no sudoeste, estão presentes em pequenas áreas de toda a Formação Boa Vista até a área dissecada do Alto Surumu, onde tornam-se mais raras. Nessas savanas os buritizais, as matas de galeria e as ilhas de matas em solos bem drenados tornam-se mais evidentes na paisagem. Savanas graminosas sem Byrsonima verbascifolia são dominantes nas Serras do Norte do Estado, mas também estão presentes nas serras do sudeste, como Serra da
Lua e em outras pequenas áreas.

Entre as savanas arbustivas e arbóreas, as savanas abertas são as mais freqüentes em Roraima, localizam-se em toda a região das savanas e ao Norte formam um mosaico com as savanas arborizadas. As savanas parques encontram-se agrupadas na região oeste, limite com a floresta estacional de transição. Essas savanas não são continuas; são pequenas ilhas, encontradas entre áreas agricolas, conservadas pelos proprietários das fazendas existentes nessa região.

As ervas e gramineas mais frequentes ( $c o m \mathrm{FR} \geq 20 \%$ ) foram Curtia tenuiflora, Cassia flexuosa, Galactia jussiaeana, Merremia aturenesis, Eriosema crinitum, Piriqueta duartiana, Stylosanthes guianensis, Trachypogon plumosus, Trachypogon spicatus, Aeschynomene paniculata, Diodia hyssopifolia, Melochia hirsuta, Aeschynomene 
Tabela 3. Características dos tipos fisionomicos das savanas de Roraima.

\begin{tabular}{|c|c|c|c|c|c|c|c|c|c|c|c|c|}
\hline \multirow{3}{*}{$\begin{array}{l}\text { Categonias } \\
N^{2} \text { de parcelas }\end{array}$} & \multicolumn{3}{|c|}{ Campo limpo } & \multicolumn{3}{|c|}{ Campo sujo } & \multicolumn{3}{|c|}{ Campo cerrado } & \multicolumn{3}{|c|}{ Savana parque } \\
\hline & \multicolumn{3}{|c|}{10} & \multicolumn{3}{|c|}{21} & \multicolumn{3}{|c|}{11} & \multicolumn{3}{|c|}{3} \\
\hline & Min. & Méd. & Max. & Min. & Méd. & Max. & Min. & Mèd. & Max. & Min. & Méd. & Max. \\
\hline$N^{2}$ arbusto/há & 0 & 6.1 & 26.7 & 13.3 & 129.8 & 406.7 & 153.3 & 207.9 & 366.7 & 433.3 & 577.8 & 693.3 \\
\hline Nª́rvore/há & 0 & 2.4 & 20.0 & 13.3 & 99.69 & 200.0 & 53.3 & 221.2 & 453.3 & 586.7 & 720.0 & 826.7 \\
\hline $\mathrm{AB} \mathrm{m} 2 / \mathrm{ha}^{*}$ & 0.003 & 1.0 & 2.8 & 1.1 & 3.4 & 8.4 & 2.7 & 4.8 & 7.3 & 5.38 & 8.79 & 11.89 \\
\hline \multicolumn{13}{|l|}{ Atura média } \\
\hline Dos ind. $>1.0 \mathrm{~m}$ & - & - & - & 1.7 & 2.7 & 4.3 & 20 & 24 & 3.0 & 2.9 & 3.0 & 3.2 \\
\hline Dos ind. $>2.0 \mathrm{~m}$ & - & & & 2.5 & 3.8 & 6.0 & 2.7 & 3.3 & 4.0 & 4.1 & 4.3 & 4.5 \\
\hline Distribuiç̧ăo** & $\cdot$ & $\cdot$ & $\cdot$ & \multicolumn{3}{|c|}{$43 \%$ agrupadas } & \multicolumn{3}{|c|}{$27 \%$ agrupadas } & \multicolumn{3}{|c|}{$100 \%$ agrupadas } \\
\hline Grau de cobertura & - & 0 & $\cdot$ & \multicolumn{3}{|c|}{$<5 \%$} & \multicolumn{3}{|c|}{ entre 2 e $15 \%$} & \multicolumn{3}{|c|}{$15 \%$} \\
\hline \multicolumn{13}{|l|}{ No de Espécies } \\
\hline Arbóreas & 0 & 0 & 1 & 1 & 4 & 9 & 1 & 4 & 12 & 5 & 9 & 14 \\
\hline Arbustivas & 0 & 0 & 2 & 2 & 4 & 8 & 2 & 5 & 10 & 8 & 10 & 13 \\
\hline Lenhosas"*. & 1 & 3 & 7 & 4 & 8 & 13 & 3 & 9 & 12 & 8 & 13 & 18 \\
\hline
\end{tabular}

* Área basal dos elementos lenhosos.

* refere-se à porcentagem de parcelas

**N Número de arbóreas, arbustivas e subarbustivas.

histrix, Axonopus aureus, Cassia hispidula, Cissampelos ovalifolia, Leptocoryphum lanatum, Borreria suaveolens, Cuphea antisyphylitica, Evolvulus sp., Rhynchospora nervosa e Sida linearifolia.

Os subarbustos mais freqüentes foram Byrsonima verbascifolia $(\mathrm{FR}=30,4 \%)$ e Tibouchina aspera $(\mathrm{FR}=17,4)$.

A maioria dos indivíduos subarbustivos foram jovens de espécies arbustivas ou arbóreas como de Casearia sylvestris, Byrsonima crassifolia, Erythroxylum suberosum, Curatella americana, Bowdichia virgilioides, Calycolpus goetheanus, Cassia obtusifolia $\mathrm{e}$ Psidium guianense.

No estrato arbustivo a densidade foi de 22,65 $\pm 24,15$ ind./parcela ( 0 104), ou seja $151 \pm 161 \mathrm{ind} . / \mathrm{ha}$. Os arbustos Curatella americana,
Byrsonima crassifolia, Byrsonima coccolobifolia, Roupala montana, Bowdichia virgilioides, Psidium guianense, Randia formosa e Xylopia aromatica foram as mais representativas desse estrato.

No estrato arbóreo a densidade foi de 22,04 $\pm 27,69$ ind./parcela (amplitude: 0 - 124), ou seja $146,9 \pm 184,6$ ind./ha. A maioria das espécies presentes no estrato arbóreo apresentaram freqüência baixa, ou seja, apresentaram-se em menos de $20 \%$ das parcelas. As árvores Curatella americana, Byrsonima crassifolia, Roupala montana, Bowdichia virgilioides, Byrsonima coccolobifolia e Xylopia aromatica foram as mais representativas.

A maioria $(84,7 \%)$ dos individuos lenhosos tinham altura de até $1,0 \mathrm{~m}$. Apenas Byrsonima 
verbascifolia, uma espécie subarbustiva, detinha $63,4 \%$ desses individuos. Muitos individuos de espécies arbustivas e arbóreas, principalmente de Curatella americana e Palicourea rigida, foram encontrados em estágio reprodutivo com menos de $50 \mathrm{~cm}$ de altura, muitas vezes com diâmetro do caule, a nivel do solo, menor que $10 \mathrm{~cm}$.

A média geral de altura dos elementos lenhosos foi de $0,8 \mathrm{~m}$ e de $2,6 \mathrm{~m}$ considerando apenas os indivíduos de árvores e arbustos. Curatella americana, Bowdichia virgilioides, Xylopia aromatica, Vitex schomburgkiana, Roupala montana e Genipa americana foram as espécies que apresentaram as árvores mais altas, entre 5 e $10 \mathrm{~m}$.
$\mathrm{Na}$ maioria $(67 \%)$ das parcelas os individuos lenhosos estavam dispersos, entretanto é comum os individuos jovens agruparem-se à sombra de grande árvores ou existir agrupamentos ao redor dos afloramentos rochosos, principalmente nas savanas do médio Rio Surumu.

Os solos de todas as parcelas mostraram $\mathrm{pH}$ fortemente ácido. Os teores de Alumínio, Magnésio, Fósforo e Matéria Orgânica são baixos, os teores de Cálcio e Sódio ocorreram em niveis médios, e os teores de potássio ocorreram em niveis altos. Os teores de areia e argila + silte foram bastante diversificados entre as 45 parcelas, apresentando grandes amplitudes (Tab. 4).

As parcelas estudadas localizamse sobre solos arenosos (19 parcelas)

Tabela 4. Tabela 8 - Média, desvio padrão e amplitude das características físicas e químicas dos solos das parcelas estudadas nas savannas de Roraima; $n=$ número de parcelas.

\begin{tabular}{|c|c|c|c|c|c|}
\hline Areia & & & Argila + Silte & & \\
\hline$n=37$ & $0-10 \mathrm{~cm}$ & $67,32 \pm 19,92(6,66-90,73)$ & $\mathrm{n}=37$ & $0-10 \mathrm{~cm}$ & $32,68 \pm 19,92(9,27-93,34)$ \\
\hline $\mathrm{pHKCl}$ & & & Alumírio & & \\
\hline$n=37$ & $0-10 \mathrm{~cm}$ & $4,23 \pm 0,30(3,80-4,98)$ & $n=37$ & $0-10 \mathrm{~cm}$ & $0,27 \pm 0,17(0,05-0,75)$ \\
\hline Cálcio & & & Mag nésio & & \\
\hline$n=33$ & $0-10 \mathrm{~cm}$ & $1,09 \pm 0,66(0,15-2,90)$ & $\mathrm{n}=32$ & $0-10 \mathrm{~cm}$ & $0,20 \pm 0,19(0,01-0,83)$ \\
\hline Potássio & & & Sódio & & \\
\hline$n=37$ & $0-10 \mathrm{~cm}$ & $1,00 \pm 0,79(0,23-2,74)$ & $\mathrm{n}=37$ & $0-10 \mathrm{~cm}$ & $0,18 \pm 0,04(0,13-0,32)$ \\
\hline Fósforo & & & Matéria Oigânica & & \\
\hline$n=35$ & $0-10 \mathrm{~cm}$ & $1,74 \pm 1,32(0,11-6,99)$ & $n=35$ & $0-10 \mathrm{~cm}$ & $1,41 \pm 0,77(0,43-2,74)$ \\
\hline
\end{tabular}

Unidades Utilizadas: Areia, Argila + Silte e $\mathrm{MO}=\%$ $\mathrm{Al}, \mathrm{Ca}, \mathrm{Mg}, \mathrm{Na}, \mathrm{K}=\mathrm{cmolc} / \mathrm{kg}$ $\mathrm{P}=\mathrm{mg} / \mathrm{kg}$ 
e solos barrentos (26 parcelas). Existe uma diferença significativa entre os valores de $\mathrm{pH}$, Magnésio, Potássio e Matéria Orgânica (Tukey p< 0.05 ) entre os solos arenosos e barentos. Os solos arenosos são um pouco mais ácidos e mais pobres em Magnésio, Potássio e Matéria Orgânica.

Oitenta por cento das parcelas classificadas como campos limpos foram encontrados sobre solos arenosos, $81 \%$ dos campos sujos sobre solos argilosos, $64 \%$ dos campos cerrados sobre solos arenosos e $100 \%$ das savanas parques foram encontradas sobre solos de areia barrenta.
Os tipos fisionômicos não possuem relações significativas com a maioria dos fatores edáficos, apenas os teores de Alumínio nas camadas de $0-10 \mathrm{~cm}$ foram diferentes significativamente entre os tipos fisionômicos $\left(\mathrm{r}^{2}=0,291 ; \mathrm{F}_{3,33}=4,509\right.$; $\mathrm{p}=0,009)$. Essa diferença significativa deve-se principalmente às diferenças encontradas entre os teores de Alumínio dos campos limpos e campos sujos ( Teste de Tukey HSD Comparações múltiplas - $\mathrm{q}_{0,05,33}=$ 11,$857 ; \mathrm{p}=0,006$ e entre os campos limpos e campos cerrados $\left(\mathrm{q}_{0,05}\right.$. ${ }_{33}=10,762 ; \mathrm{p}=0,022$ ) (Fig. 1).

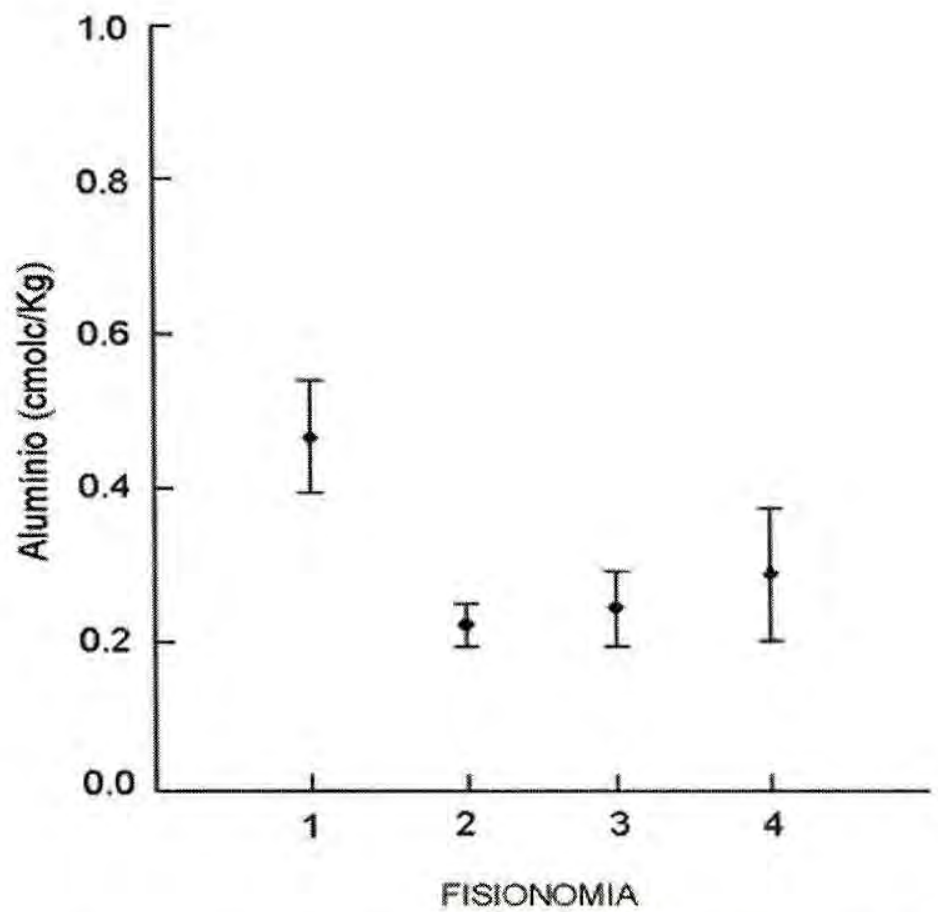

Figura 1. Teores de Alumínio dos solos superficiais $(0-10 \mathrm{~cm}$ de profundidade) dos quatro tipos físionômicos ( $1=$ campo limpo; 2 = campo sujo; $3=$ campo cerrado; $4=$ savana parque $)$. Os pontos representam as médias e as linhas verticais os erros padrões. 


\section{DISCUSSÃO}

Nas savanas Neotropicais inicialmente pode-se diferenciar dois estratos, o herbáceo, geralmente contínuo, e o lenhoso, geralmente com árvores e/ou arbustos dispersos (Monasterio \& Sarmiento, 1968; Sarmiento \& Monasterio, 1971). Nas savanas de Roraima podem facilmente ser identificados três estratos: herbáceo, arbustivo e arbóreo.

A maioria das ervas e dos subarbustos que compõem o estrato herbáceo foram encontrados em poucas parcelas, sendo consideradas espécies raras. Essas espécies ou possuem exigências de habitat diferentes ou se excluem devido a competição (Noy-Meir \& Maarel, 1987). As gramineas mais frequentes são amplamente distribuídas nas savanas do norte da América do Sul e as ciperáceas são muito mais frequentes em Roraima do que nos cerrados do Brasil Central mas, como mostra Miranda \& Absy (1997) sua biomassa, em Roraima, é muito menor do que a biomassa das gramíneas, ao contrário do que sugere Vanzolini \& Carvalho (1991).

As savanas graminosas não possuem estrato lenhoso acima do herbáceo, mas podem ser puras (encontradas principalmente na região serrana do norte do Estado, constituindo as savanas estépicas graminosas descritas por Veloso et al. (1975) ou podem possuir arbustos ou árvores anãs, principalmente Byrsonima verbascifolia uma árvore típica dos cerrados brasileiros que pode alcançar até $4,0 \mathrm{~m}$ de altura no Planalto Central (Pagano et al., 1989).

As espécies arbustivas e arbóreas geralmente estão também nos estratos inferiores, como jovens e às vezes como adultos. Segundo Sarmiento (1984) é comum encontrarmos adultos de lenhosas no estrato herbáceo, ou devido ao fogo ou às condições adversas ao crescimento vegetativo.

A média de altura das árvores $(2,60 \mathrm{~m})$ das savanas de Roraima é comparável à altura relatada por Takeuchi (1960) para os campos de Roraima - entre 2,0 e 3,0 m. Entretanto é um pouco mais baixa do que a altura dos cerrados brasileiros. Segundo Eiten (1977) e Silberbauer-Gottsberger \& Eiten (1987) a altura média das árvores do cerrado é de $3,0 \mathrm{~m}$.

Goodland (1971) verificou um gradiente de altura arbórea entre os campos sujos e o cerradão do Triângulo Mineiro no sudeste do Brasil (altura média dos campos sujos $=3,0 \mathrm{~m}$; dos campos cerrados $=4,0$ $\mathrm{m} ;$ dos cerrados sensu stricto $=6,0 \mathrm{~m}$; e, do cerradão $=9,0 \mathrm{~m})$. A média de altura $(3,8 \mathrm{~m}$ para ind. $>2,0 \mathrm{~m})$ encontrada nas savanas abertas (ou campos sujos) de Roraima foi maior do que a média de altura $(3,3 \mathrm{~m}$ para ind. $>2,0 \mathrm{~m}$ ) encontrada nas savanas arborizadas (ou campos cerrados) e comparável à média de altura encontrada na savana parque $(4,3 \mathrm{~m}$ para ind. $>2,0 \mathrm{~m}$ ).

Portanto, não foi encontrado um gradiente de altura média em Roraima, devido principalmente às diferentes porcentagens de indivíduos nas classes de altura. Porém a densidade de 
árvores $>2,0 \mathrm{~m}$ segue um gradiente das savanas abertas para as savanas arborizadas e savanas parques.

A distribuição espacial das árvores e arbustos nas savanas neotropicais geralmente segue dois padrões: um com os elementos lenhosos bem espalhados pelo contínuo estrato herbáceo e o outro com os elementos lenhosos agrupados (Sarmiento, 1984). Esses dois padrões foram identificados nas savanas de Roraima.

A distribuição agrupada foi encontrada principalmente na savana parque, localizadas na área da Formação Boa Vista e nas savanas abertas e savanas arborizadas da superfície dissecada do médio Surumu.

Segundo Hoffmann (1996) a cobertura arbórea favorece o estabelecimento de plântulas nos cerrados de Brasilia-DF. Os solos sob árvores das savanas tem melhores teores de nutrientes (Kellman, 1986; Belsky et al., 1989; Isichei \& Muoghalu, 1992), maior umidade durante os períodos secos e apresenta uma redução da temperatura superficial (Belsky et al., 1989), originando um diferente microhabitat capaz de modificar os estratos inferiores (Sarmiento, 1984).

$\mathrm{O}$ agrupamento em moitas e as altas densidades, área basal e grau de cobertura foram os critérios utilizados para classificar essas parcelas como savana parque. Apesar de serem encontradas parcelas que apresentaram agrupamentos em moitas nas fisionomias de savana aberta e savana arborizada, as densidades de árvores e arbustos, a área basal e a cobertura arbórea foram mais importantes nas suas classificações. Segundo Sarmiento (1984) essas fisionomias podem ou não apresentar agrupamentos em moitas.

A maioria das caracteristicas fisionômicas analisadas nas savanas de Roraima seguem um gradiente das savanas graminosas para a savana parque, comparável aos gradientes encontrados por Goodland (1971) e propostos por Eiten (1972) e Coutinho (1978) para os cerrados brasileiros.

O mosaico de tipos fisionômicos encontrado neste trabalho, não confirma a distribuição geográfica dos tipos fisionômicos estabelecida por Veloso et al. (1975), onde as fisionomias distribuem-se em diferentes regiões geográficas das savanas de Roraima. Com exceção de pequenos locais, como as savanas graminosas com Byrsonima verbascifolia e savanas estépicas graminosas que predomina numa região geográfica, os tipos fisionômicos formam um mosaico de vegetação, podendo ser encontrados em diferentes regiões.

Assim como os cerrados do Brasil Central e outras savanas neotropicais os solos das savanas de Roraima mostraram fortemente ácidos e pobres em nutrientes e matéria orgânica (Goodland \& Pollard, 1973; Lopes \& Cox, 1977). Entretanto as médias de Aluminio foram baixas quando comparadas com os níveis encontrados por Haridasan (1982) nos cerrados de Brasília e Goodland \& 
Ferri (1979) nos cerrados do Triângulo Mineiro, apesar da grande variação nos teores de Aluminio dos solos das savanas de Roraima.

Dantas \& Rodrigues (1982), analisando o solo superficial de cinco diferentes localidades nas savanas de Roraima, também encontraram baixos teores de fósforo e matéria orgânica e altos teores de potássio; entretanto os níveis de Alumínio foram bem superiores do que os níveis apresentados neste trabalho.

Os solos arenosos encontrados nas savanas de Roraima foram, em geral, mais ácidos na camada superficial e com menores teores de potássio e Magnésio do que os solos barrentos. Segundo Freitas \& Silveira (1977) os solos arenosos, em geral, são profundos, fortemente ácidos, com permeabilidade rápida, destituído de minerais primários que são facilmente decompostos.

A textura dos solos e os teores de Alumínio, que apresentaram-se mais elevados nos campos limpos, podem estar determinando a fisionomia das savanas de Roraima, embora outros fatores bióticos e abióticos, que aqui não foram estudados, como altitude, clima e fogo podem também ser importantes para a fisionomia.

\section{AGRADECIMENTOS}

O trabalho foi financiado pelo programa ECOFIT (ORSTOM) e recebeu apoio do Núcleo de Pesquisas do INPA em Roraima (INPA/NPRR), da Coordenação de Pesquisas em Botânica

(CPBO/INPA)
Coordenação de Pesquisas em Ciências Agronômicas (CPCA/INPA). Agradecemos a George H. Rebêlo e Thyerry Desjardins pelas críticas e sugestões.

\section{Bibliografia Citada}

Ambtec. 1994. Roraima. O Brasil do Hemisfério Norte: Diagnóstico Científico e Tecnológico para o Desenvolvimento. Fundação do Meio Ambiente e Tecnologia de Roraima, Boa Vista, 512p.

Belsky, A.J.; Amundson, R.G.; Duxbury, J.M.; Riha, S.J.; Ali, A.R.; Mwong, S.M. 1989. The effects of trees on their physical, chemical, and biological environments in a semi-arid savanna in Kenya. Journal of Applied Ecology, 26:1005-1024.

Borhidi, A. 1988. Vegetation dynamics of the savannization process on Cuba. Vegetatio, $77: 177-183$.

Camargo, A.P.; Alfonsi, R.R.; Pinto, H.S.; Chiarini, J.V. 1976. Zoneamento da aptidão climática para culturas comerciais em áreas de cerrado. In: Ferri, M.G. (Cood.) IV Simpósio sobre o cerrado. Itatiaia, EDUSP, São Paulo. p.89-120.

Correa, P.R.S.; Peres, R.N.; Souza, L.F.P. 1975. Pedologia. Levantamento exploratório. In: BRASIL. Projeto RADAMBRASIL. Folha, NA. 20 Boa Vista e parte das Folhas NA. 21 Tumucumaque, NB. 20 Roraima e NB. 21. DNPM, Ministério das Minas e Energia. Departamento Nacional da Produção Mineral. Rio de Janeiro. p.181305.

Coutinho, L.M. 1978. O conceito do Cerrado. Revista Brasileira de Botânica, 1:17-23.

Dantas, M.; Rodrigues, A.I. 1982. Estudos Fitoecológicos do Trópico úmido Brasileiro: IV- Levantamentos Botânicos em Campos do Rio Branco. EMBRAPA/ CPATU, Boletim de Pesquisa, 40:1-31.

Durigan, G.; Leitão Filho, H.F.; Rodrigues, R.R. 1994. Phytosociology and structure of a frequently burnt cerrado vegetation in 
SE-Brazil. Flora, 189:153-160.

Eiten, G. 1972. The cerrado vegetation of Brazil. The Botanical Review, 38:201-341.

Eiten, G. 1977. Delimitação do conceito de cerrado. Arquivos Jardim Botânico do Rio de Janeiro, 21:125-134.

Eiten, G. 1983. Brazilian 'savannas'. In: Huntley, B.J.; Walker, B.H. (Eds.) Ecology of Tropical Savannas. Springer Verlag, Berlim. p.25-47.

Franco, E.M.S.; Del'Arco, J.O.; Rivetti, M. 1975. Geomorfologia. In: BRASIL. Projeto RADAMBRASIL, Folha, NA. 20 Boa Vista e parte das Folhas NA. 21 Tumucumaque, NB. 20 Roraima e NB. 21. DNPM, Ministério das Minas e Energia. Departamento Nacional da Produção Mineral. Rio de Janeiro, p.137-180.

Freitas, F.G.; Silveira, C.O. 1977. Principais solos sob a vegetação de cerrado e sua aptidão agrícola. In: Ferri, M.G. (Coord.) IV Simpósio Sobre o Cerrado. EDUSP, Ed. Itatiaia, São Paulo. p.155-195.

Furley, P.A.; Ratter, J.A. 1988. Soil resources and communities of the Central Brazilian cerrado and their development. Journal of Biogeography, 15:97-108.

Gatto, L.C.S. 1991. Relevo. In: IBGE, Geografia do Brasil: Região Norte, Vol. 3. IBGE, Rio de Janeiro. p.47-71.

Gibbs, P.E.; Leitão Filho, H.F.; Shepherd, G.J. 1983. Floristic Composition and community structure in an area of cerrado in SE Brazil. Flora, 173:433-449.

Goldsmith, F.B. 1974. Multivariate analysis of tropical grassland communities in Mato Grosso, Brazil. Journal of Biogeography, $1: 111-122$.

Goodland, R. 1971. A physionomic analysis of the cerrado vegetation of Central Brazil. Journal of Ecology, 59:411-419.

Goodland, R.; Pollard, R. 1973. The brazilian cerrado vegetation: a fertility gradient. Journal of Ecology, 61:219-224.

Goodland, R.; Ferri, M.G. 1979. Ecologia do Cerrado, EDUSP, São Paulo. 193p.

Haridasan, M. 1982. Aluminium accumulation by some cerrado native species of cen- tral Brazil. Plant and Soil, 65:265-273.

Hoffmann, W.A. 1996. The effects of fire and cover on seedling establishment in a neotropical savanna. Journal of Ecology, 84:383-393.

Huber, O. 1982. Significance of savanna vegetation in the Amazon Territory of Venezuela. In: Prance, G.T. (ed.) Biological Diversification in the Tropics. Columbia University Press, New york. p.221-244.

Isichei, A.O.; Muoghalu, G.I. 1992. The effects of tree canopy cover on soil fertility in a Nigerian savanna. Journal of Tropical Ecology, 8:329-338.

Kellman, M, 1986. Fire Sensitivity of Casuarina torulosa in North Queensland, Australia. Biotropica, 18:107-110.

Lopes, A.S.; Cox, F.R. 1977. Cerrado vegetation in Brazil: an edaphic gradient. Agronomy Journal, 69:828-831.

Medina, E. 1987. Nutrients: requirements, conservation and cycles in the herbaceous layer. In: Walker, B.W. (ed.) Determinants of savannas. IUBS monograph series no. 3. IRL Press, Oxford. p.39-67.

Miranda, I.S. 1998. Flora, Fisionomia e Estrutura das Savanas de Roraima. Brasil. Tese de Doutoramento, Instituto Nacional de Pesquisas da Amazônia/ Universidade do Amazonas, Manaus, Brasil. 186p.

Miranda, I.S.; Absy, M.L. 1997.: Flora Fanerogâmica das Savanas de Roraima. In: Barbosa, R.I.; Ferreira, E.J.G.; Castellõn, E.G. Homem, Ambiente e Ecologia no Estado de Roraima. INPA, Manaus. p.445-462.

Monasterio, M.; Sarmiento, G. 1968. Análisis ecológico y fitosociológico de la sabana en la Estación Biológica de los Llanos. Boletim da Sociedade Venezuelana de Ciências Naturais., 113:477-524.

Montgomery, R.F.; Askew, G.P. 1983. Soils of tropical savannas, In: Bourliere, F. (Ed.) Tropical Savannas Ecosystems of the World. Elsevier, Amsterdam. p.63-78.

Nimer, E. 1991. Clima. In: IBGE, Geografia 
do Brasil: Região Norte, Vol. 3. IBGE, Rio de Janeiro. p.61-71.

Noy-Meir, I.; Maarel, E. van der. 1987. Relations between community theory and community analysis in vegetation science: some historical perspectives. Vegetatio, 69:5-15.

Oliveira, A.T.; Martins, F.R. 1986. Distribuição, caraterização e composição floristica das formações vegetais da região da salgadeira, na Chapada dos Guimarães (MT). Revista Brasileira de Botânica, 9:207-223.

Oliveira, A.T.; Shepherd, J., Martins, F.R.; Stubblebine, W.H. 1989. Environmental factors affecting physiognomic and floristic variation in an area cerrado in central Brazil. Journal of Tropical Ecology, 5:413-431.

Pagano, S.N.; Cesar, O.; Leitão Filho, H.F. 1989. Estrutura Fitossociológica do Estrato Arbustivo-Arbóreo da Vegetação de Cerrado da Área de Proteção Ambiental (APA) de Corumbataí - Estado de São Paulo. Revista Brasileira de Biologia, 49:49-59.

Pires, J.M.; Prance, G.T. 1985. The Vegetation Types of the Brazilian Amazon, In: Prance, G.T.; Lovejoy, T.E (Eds.) Key Environments Amazonia. Pergamon Press, Oxford. p.109-145.

Prance, G.T. 1996. Islands in Amazonia. Philosophical Transactions of the Royal Society, London, 351:823-833.

Queiroz Neto, 1982. Solos da região dos cerrados e suas e suas interpretações. Revista Brasileira de Ciencia do Solo, $6: 1-12$.

Ratter, J.A.; Dargie, T.C.D. 1992. An analysis of the floristic composition of 26 cerrado areas in Brazil. Journal of the Edinburgh Botanical Garden, 49:235-250.

Sarmiento, G. 1984. The Ecology of Neotropical Savannas. Haward University Press. Cambrige. 235p.
Sarmiento, G.; Monasterio, M. 1971. Ecologia de las sabanas de América Tropical. I. Análisis macro ecológica de los Llanos de Calabozo, Venezuela. Cuadernos Geográficos, 4:1-126.

Sarmiento, G.; Monasterio, M. 1975. A critical consideration of the enviromental conditions associated with the occurence of savanna ecosystems. In: Golley, F.B. Medina, E. (Eds.) Tropical Ecological Systems. Spring-Verlag, New York. p.223250.

Silberbauer-Gottsberger, I. ; Eiten, G. 1987. A Hectare of Cerrado. I. General Aspects of the Trees and Thick-Stemmed Shrubs. Phyton, 27:55-91.

Takeuchi, M. 1960. A Estrutura da Vegetação na Amazônia. II- As Savanas do Norte da Amazônia. Boletim do Museu Paraense Emilio Goeldi, série Botânica, 7:1-14.

Vanzolini, P.E.; Carvalho, C.M. 1991. Two sibling and sympatric species of Gymnophthalmus in Roraima, Brasil (Sauria, Teiidae). Papéis Avulsos de Zoologia, USP, 37:173-226.

Veloso, H.P.; Goes Filho, L.; Leite, P.F.; Silva, S.B.; Ferreira, H.C.; Loureiro, R.L.; Terezo, E.F.M. 1975. Vegetação: as regiões fitoecológicas, sua natureza e seus recursos econômicos - estudo fitogeográfico. In: BRASIL. Projeto RADAMBRASIL. Folha, NA. 20 Boa Vista e parte das Folhas NA. 21 Tumucumaque. NB. 20 Roraima e NB. 21. DNPM, Ministério das Minas e Energia. Departamento Nacional da Produção Mineral. Rio de Janeiro. p.305-335.

Veloso, H.P.; Rangel Filho, A.L.R.; Lima, J.C.A. 1991. Classificação da Vegetação Brasileira, Adaptada a um Sistema Universal. IBGE, Rio de Janeiro. 123p.

Zar, J.H. 1984. Biostatistical Analysis. Prentice Hall international, New Jersey. 718p. 